\section{AUF1 promotes let-7b loading on Argonaute 2}

\author{
Je-Hyun Yoon, 1,7 Myung Hyun Jo, 2,7 \\ Elizabeth J.F. White, ${ }^{3}$ Supriyo De, ${ }^{1}$ Markus Hafner, ${ }^{4}$ \\ Beth E. Zucconi, ${ }^{3}$ Kotb Abdelmohsen, ${ }^{1}$ \\ Jennifer L. Martindale, ${ }^{1}$ Xiaoling Yang, ${ }^{1}$ \\ William H. Wood III, ${ }^{1}$ Yu Mi Shin, ${ }_{1}^{5}$ Ji-Joon Song, ${ }^{5}$ \\ Thomas Tuschl, ${ }^{6}$ Kevin G. Becker, ${ }^{1}$ \\ Gerald M. Wilson, ${ }^{3}$ Sungchul Hohng, ${ }^{2}$ \\ and Myriam Gorospe ${ }^{1}$
}

${ }^{1}$ Laboratory of Genetics, National Institute on Aging-Intramural Research Program, National Institutes of Health, Baltimore, Maryland 21224, USA; ${ }^{2}$ Department of Physics and Astronomy, Institute of Applied Physics, National Center for Creative Research Initiatives, Seoul National University, Seoul 151-747, Korea; ${ }^{3}$ Department of Biochemistry and Molecular Biology, Marlene and Stewart Greenebaum Cancer Center, University of Maryland School of Medicine, Baltimore, Maryland 21201, USA; ${ }^{4}$ Laboratory of Muscle Stem Cells and Gene Regulation, National Institute of Arthritis and Musculoskeletal and Skin DiseasesIntramural Research Program, National Institutes of Health, Bethesda, Maryland 20892, USA; ${ }^{5}$ Cancer Metastasis Control Center, Institute for the BioCentury, Korea Advanced Institute of Science and Technology (KAIST), Daejeon 305-338, Korea;

${ }^{6}$ Howard Hughes Medical Institute, Laboratory of RNA

Molecular Biology, Rockefeller University, New York, New York 10065, USA

Eukaryotic gene expression is tightly regulated post-transcriptionally by RNA-binding proteins (RBPs) and microRNAs. The RBP AU-rich-binding factor 1 (AUF1) isoform p37 was found to have high affinity for the microRNA let-7b in vitro $\left(K_{d}=\sim 6 \mathrm{nM}\right)$ in cells. Ribonucleoprotein immunoprecipitation, in vitro association, and single-molecule-binding analyses revealed that AUF1 promoted let-7b loading onto Argonaute 2 (AGO2), the catalytic component of the RNA-induced silencing complex (RISC). In turn, AGO2-let-7 triggered target mRNA decay. Our findings uncover a novel mechanism by which AUF1 binding and transfer of microRNA let-7 to AGO2 facilitates let-7-elicited gene silencing.

Supplemental material is available for this article.

Received April 12, 2015; revised version accepted July 16, 2015.

Given that RNA-binding proteins (RBPs) are potent posttranscriptional regulators of gene expression (Glisovic et al. 2008), there is great interest in identifying comprehensively the functions of RBPs. The RBP AU-binding factor 1 (AUF1) comprises four isoforms-p37, p40, p42,

[Keywords: Argonaute; RISC; hnRNP D; microRNA; ribonucleoprotein complex]

${ }^{7}$ These authors contributed equally to this work.

Corresponding authors: yoonjehyun@gmail.com, shohng@snu.ac.kr

Article is online at http://www.genesdev.org/cgi/doi/10.1101/gad.263749. 115 . and p45-and has been closely linked to physiological and pathological processes, including cellular senescence, myogenesis, inflammation, aging, and cancer(Zucconi and Wilson 2011; Pont et al. 2012; Panda et al. 2014). Although all isoforms contain two RNA recognition motifs (RRMs) followed by a glutamine-rich domain, p37 and p40 lack exon 7, while p37 and p42 lack exon 2. The four isoforms can bind to similar RNA sequences but display distinct subcellular localization profiles, affinities for target mRNAs, and impacts on the fate of mRNA targets (White et al. 2013). We recently used the method PAR-CLIP (photoactivatable ribonucleoside-enhanced cross-linking and immunoprecipitation) (Hafner et al. 2010, 2012) to identify comprehensively the RNA targets of AUF1 (Yoon et al. 2014). The analysis revealed that AUF1 primarily recognized U/GU-rich sequences in target mRNAs and long noncoding RNAs (lncRNAs) and was capable of destabilizing some target RNAs, as previously described (Zucconi and Wilson 2011; Pont et al. 2012), but also stabilized other target transcripts and altered the translation of a subset of mRNAs (Yoon et al. 2014). By increasing the expression of several target $\mathrm{mRNAs}$ encoding genomic maintenance proteins, AUF1 was found to help preserve DNA integrity; this function is in keeping with the ability of AUF1 to prevent premature cellular senescence and aging (Pont et al. 2012; White et al. 2013; Yoon et al. 2014).

Earlier evidence indicated a functional connection between AUF1 and Argonaute (AGO) proteins, the catalytic components of the RNA-induced silencing complex (RISC) (Chang et al. 2010; Wu et al. 2013). The main function of the argonaute proteins is to bind microRNAs, a family of short ( 22-nucleotide [nt]) noncoding RNAs that form partial hybrids with target mRNAs, often through the "seed" region (nucleotides 2-7) of the microRNA (Ambros 2004; Bartel 2004). AGO-microRNA complexes in turn recruit the RISC to an mRNA and silence the mRNA by reducing its stability and/or translation (Filipowicz 2005; Joshua-Tor 2006; Chekulaeva and Filipowicz 2009). Here, we report that AUF1 p37 displayed strong affinity for let-7b, enhanced the loading of let-7b on AGO2 in vitro, and promoted AGO2-let-7-mediated mRNA decay. Our findings underscore a new mechanism by which microRNA transfer from AUF1 p37 to AGO2 facilitates microRNA-elicited gene silencing.

\section{Results and Discussion}

\section{AUF1 binds let-7b with high affinity}

During earlier studies to investigate HuR interaction with microRNAs (Yoon et al. 2013), we observed that AUF1 was also capable of binding microRNAs, although the AUF1-microRNA interactions did not meet the stringent criteria of being PAR-CLIP targets. We used fluorescence anisotropy-based binding assays to investigate in detail the binding of purified recombinant, His-tagged AUF1 p37, p40, p42, and p45 (Fig. 1A, right) to study two of these

(C) 2015 Yoon et al. This article is distributed exclusively by Cold Spring Harbor Laboratory Press for the first six months after the full-issue publication date (see http://genesdev.cshlp.org/site/misc/terms.xhtml). After six months, it is available under a Creative Commons License (Attribution-NonCommercial 4.0 International), as described at http:// creativecommons.org/licenses/by-nc/4.0/. 

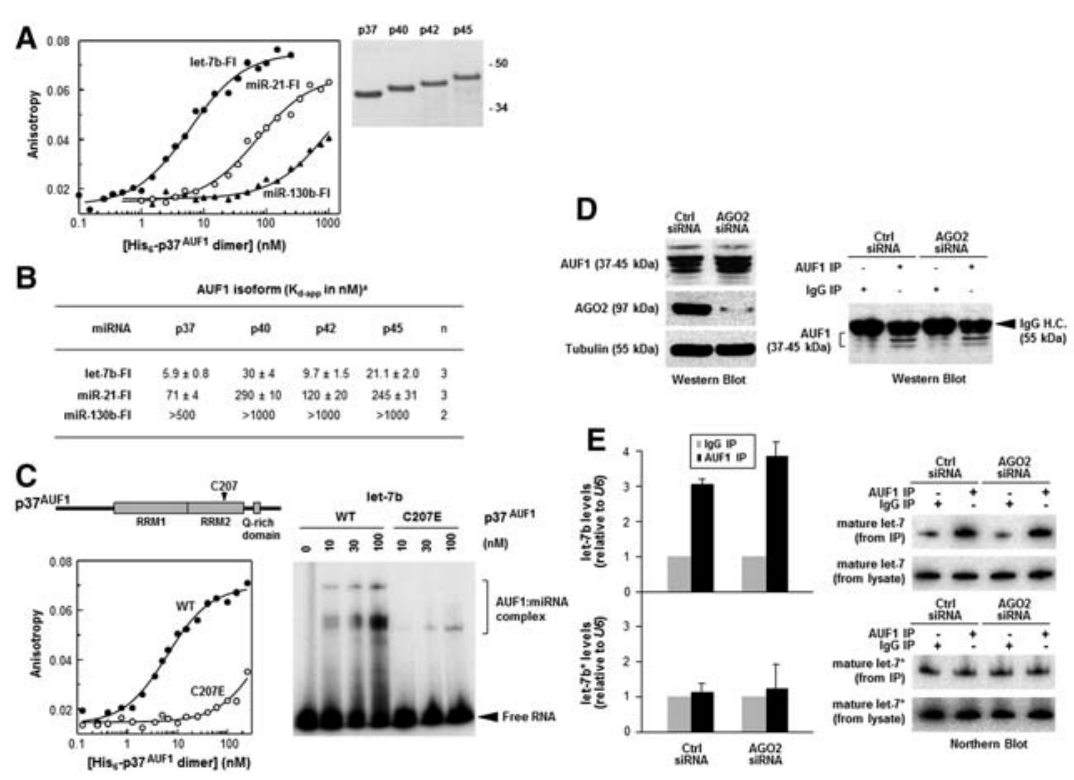

Figure 1. AUF1 binds microRNA let-7b. (A) In vitro binding of recombinant AUF1 p 37 to fluorescein-tagged let-7b, miR-21, and $\mathrm{miR} 130 \mathrm{~b}$ was analyzed by fluorescence anisotropy. (Right) Gel analysis (Coomassie) of the AUF1 isoforms tested. RNP complex affinity of AUF1 p37 was resolved using a single-site-binding model. $(B)$ The table lists the affinities of AUF1 binding to microRNA substrates; values are the apparent equilibrium dissociation constants $\left(K_{\mathrm{d} \text {-app }}\right)$ describing AUF1 dimer binding to each microRNA target resolved using Equation 1 (Supplemental Material). Binding constants represent the mean \pm SD of $n$ independent isotherms. (C) Schematic representation of wild type (AUF1 WT) and a mutant unable to bind RNA(AUF1 C207E). (Left) RNP complex affinity of AUF1 p37 wild type and C207E resolved using a single-site-binding model. (Right) RNA EMSA of radiolabeled let-7b with recombinant AUF1 p37 and mutant C207E. (D) Forty-eight hours after transfecting HeLa cells with control (Ctrl) siRNA or AGO2 siRNA, Western blot analysis was used to assess the steady-state levels of AUF1, AGO2, and loading control Tubulin (top left) as well as the levels of immunoprecipitated AUF1 (top right). (IgG H.C.) Immunoglobulin heavy chain. (E) In the transfection groups described in D, AUF1let-7b complexes (and negative control AUF1-let-7b* complexes) were analyzed by AUF1 ribonucleoprotein immunoprecipitation (RIP) followed by detection of let-7b levels by RT-qPCR (left) and Northern blot (right) analyses. Data in $A, C$, and $D$ are representative of three independent experiments; data in $E$ are the means \pm SD of three independent experiments.

microRNAs (let-7b and miR-21) that contained UU- and UG-rich sequences characteristic of AUF1 targets (Yoon et al. 2014). A control microRNA that did not bind AUF1, miR-130b, was also included. Fluorescein-tagged let-7b formed direct complexes with AUF1, consistent with a single-site-binding model (Fig. 1A; Supplemental Fig. S1A), with particularly low $K_{\mathrm{d}}$ values for p37 (5.9 nM) and p42 (9.7 nM) (Fig. 1B). Notably, AUF1 p37 had significantly stronger affinity for let-7b than for a similarly sized (20-nt) AU-rich element from the TNFa mRNA 3' untranslated region (UTR), a hallmark substrate for AUF1 $\left(K_{\mathrm{d}}=33 \mathrm{nM}\right)$ (Supplemental Fig. S1B; Zucconi et al. 2010). In contrast, the affinities of AUF1 p37 for miR-21 and miR-130b were weaker (Fig. 1A,B; Supplemental Fig. S1A-C), and AUF1 isoforms p45 and p40 displayed relatively lower affinity for all three microRNAs (Fig. 1A,B; Supplemental Fig. S1A). We confirmed the interaction of AUF1 with the main target, let-7b, by using RNA electrophoretic mobility shift assay (EMSA) (Fig. 1C). As shown, a mutant AUF1 unable to bind RNA due to a C-to-E mutation at position 207 within the second RRM (RRM2) did not bind let-7b, let- $7 b^{*}$, or the canonical substrate (Fig. 1C; Supplemental Fig. S1B,D,E).

Next, we tested whether let-7b interacted with endogenous AUF1 in HeLa (human cervical carcinoma) cells by using ribonucleoprotein immunoprecipitation (RIP) anal- ysis. Detection of let-7 by Northern blot analysis and RT-qPCR analysis of small RNAs (Yoon et al. 2012a) revealed that mature let-7 microRNA was enriched in AUF1 immunoprecipitation samples but not in control IgG immunoprecipitation samples, further indicating that endogenous AUF1 is capable of interacting with endogenous mature let-7 (Fig. 1D). These results also showed that AUF1 binding to let-7b did not depend on the levels of the main protein that binds mature microRNAs, AGO2, as silencing AGO2 did not change let-7 enrichment in AUF1 immunoprecipitation samples (Fig. 1E). Binding of AUF1 to miR-21 and miR-130b by RIP and RT-qPCR analysis was also unaffected by AGO2 levels; importantly, the levels of these microRNAs was unchanged (Supplemental Fig. S1F,G). Recombinant purified AGO2 and AUF1 p37 showed interaction in vitro (Supplemental Fig. S1H).

\section{AUF1 facilitates 1et-7b loading onto AGO2}

We then tested the reciprocal possibility that silencing AUF1 might affect AGO2 interaction with let-7b in the context of the cell. After silencing AUF1 in HeLa cells (Fig. 2A), we measured let-7b bound to AGO2 by RIP followed by RT-qPCR analysis of small RNAs (Yoon et al. 2012a). Our results show that AGO2 binding to let-7b was reduced when AUF1 was silenced (Fig. 2B), supporting the notion that AUF1 facilitated the AGO2-let-7b interaction; importantly, silencing AUF1 did not affect let-7b levels (Fig. 2C). Mouse embryo fibroblasts (MEFs) with homozygous deletion of the Auf1(Hnrnpd) gene (Pont et al. 2012) also showed severely reduced interaction of $\mathrm{AGO} 2$ with let$7 \mathrm{~b}$ and no changes in the levels of let-7b or other microRNAs (Fig. 2D). Despite being weaker targets of AUF1, binding of miR-21 and miR-130b to AGO2 was also reduced when AUF1 was silenced or knocked out, but AGO2 binding to miR-10 (not a target of AUF1) was unaffected (Supplemental Fig. S2). In vitro binding assays further supported this hypothesis, since the interaction of AGO2 (His-AGO2) with let-7b was enhanced in the presence of recombinant His-tagged AUF1 p37 (His-AUF1) but not His-tagged AUF1 p37 C207E (Fig. 2E). These findings indicated that the interaction of AGO2 with let-7b was impaired when AUF1 levels were substantially reduced.

To study more directly the result that AUF1 influenced AGO2 binding to let-7b, we used a single-molecule-binding assay (Jo et al. 2015; Salomon et al. 2015). We tested whether AUF1 might compete with AGO2 for binding let-7b or instead was capable of transferring let-7b to AGO2 (Fig. 3A-C; Supplemental Fig. S3). Internally labeled (Cy5) let-7b (100 pM) was incorporated onto AGO2 immobilized to a glass slide dynamically at a rate of $\sim 190$ molecules per second per micromole (Fig. 3D; 


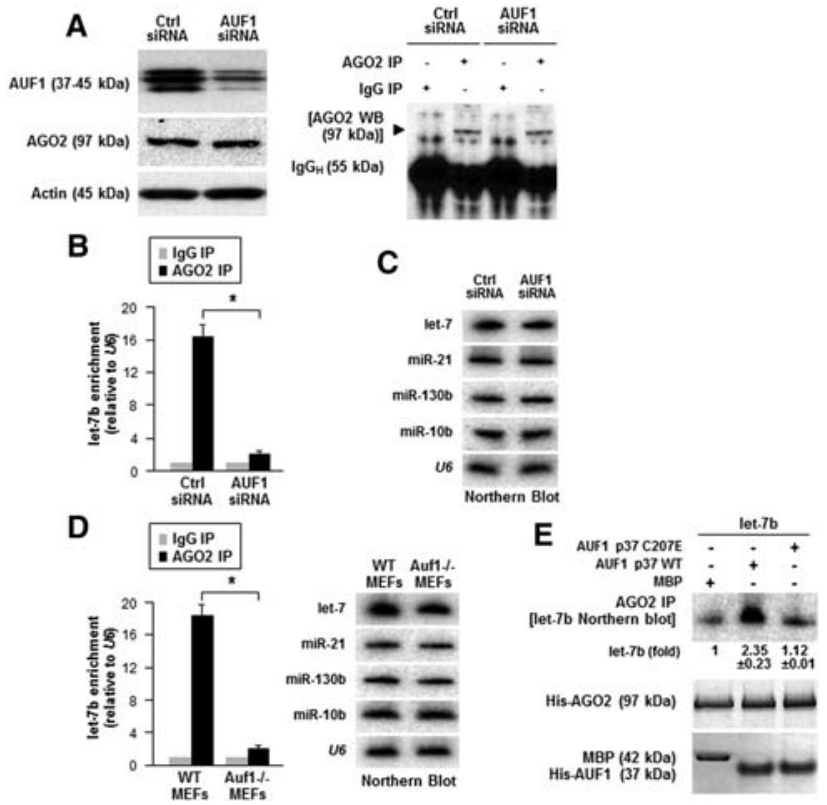

Figure 2. AUF1 promotes AGO2-microRNA interactions. $(A) \mathrm{HeLa}$ cells were transfected with either AUF1-directed siRNA or control (Ctrl) siRNA and analyzed $48 \mathrm{~h}$ later. The steady-state levels of AUF1, AGO2, and housekeeping protein Actin as well as the levels of AGO2 in the immunoprecipitation (IP) samples were assessed by Western blot analysis. $(B, C)$ Forty-eight hours after transfecting HeLa cells with the siRNAs shown in $A$, let-7b-AGO2 associations were assessed by RIP analysis $(B)$, and the total levels of let-7b and other microRNAs relative to loading control U6 were assessed by Northern blot analysis $(C)$. (D) RIP analysis of AGO2 interaction with let-7b (left) and Northern blot analysis of the steady-state levels of the microRNAs shown (right) were carried out in wild-type (WT) and Auf1 ${ }^{-1-}$ MEFs. (E) Binding of let-7b to purified recombinant AGO2. Following incubation and immunoprecipitation, the abundance of let-7b was assessed by Northern blot analysis, and the proteins in the reaction were visualized using Coomassie blue stain (AGO2, MBP, AUF1 p37, and AUF1 p37 C207E). Data in $A, C, D$, and $E$ are representative of three independent experiments; data in $B$ and $D$ represent the means \pm SD of three independent experiments.

Supplemental Fig. S3A). If AUF1 competed with AGO2 for binding let-7b, Cy5-let-7b prebound to recombinant AUF1 p37 (at an excess concentration of $100 \mathrm{nM}$, thus ensuring that all of let-7b was bound to 337 AUF1) would not be transferred to AGO2. Instead, AUF1 was found to transfer Cy5-let-7b to AGO2 at a rate of 126 molecules per second per micromole (Fig. 3D). This rate of transfer was only slightly slower when let-7b and AUF1 p37 were added at the same time without preloading $(94$ molecules per second per micromole) (Fig. 3D), further indicating that AUF1 and AGO2 did not compete for binding to let-7b and supporting the enhanced levels of AGO2-let-7b complexes in the presence of AUF1 (Fig. 2). As controls, HuR did not transfer let-7b to AGO2 at all, and AUF1 did not transfer another microRNA (miR-21) to AGO2 (Fig. 3D).

To test the loading efficiencies of let-7b to AGO2, we compared Cy5-let-7b signals $10 \mathrm{~min}$ after injection of let- $7 \mathrm{~b}$, reflecting binding events that had occurred between 0 and $600 \mathrm{sec}$ of exposure to Cy5-let-7b. As shown, without AGO2 immobilization, virtually no Cy5-let-7b was bound to the glass slide regardless of the presence of AUF1 p37, but, in the presence of immobilized AGO2, there were numerous Cy5-let-7b spots whether or not p37 AUF1 was present (Fig. 3E). Other AUF1 isoforms that bound let-7b with lower affinity (p40, p42, and p45) (Fig. 1; Supplemental Fig. S1A) did not transfer Cy5-let$7 \mathrm{~b}$ to AGO2 efficiently (Supplemental Fig. S3B,C). As a further control, immobilization of human HILI (hHILI), a member of the AGO/PIWI family that associated with small RNAs (piwi-RNAs) but not microRNAs, did not bind Cy5-let-7b alone or when complexed with AUF1 p37 (Supplemental Fig. S3D). Finally, to gain further insight into the AUF1-let-7b complex that yields let-7b to AGO2, we treated let-7b alone and AUF1-let-7 complexes with RNase A before loading them onto AGO2 slides; as shown in Figure 3E, AUF1 effectively protected let-7b from degradation by RNase A treatment and successfully yielded ("passed on") let-7b to AGO2. Together, the single-molecule experiments indicate that microRNA let$7 \mathrm{~b}$ can be mobilized from AUF1 p37 to AGO2 and suggest that AUF1 p37 might protect microRNAs in transit to AGO2. In sum, the results obtained using a variety of binding experiments-single-molecule experiments, intact cell assays, assays in cell lysates, and in vitro experiments-all support a model in which AUF1 enhances the formation of AGO2-let-7 complexes.

\section{AUF1-enhanced AGO2-let-7b interaction promotes let-7b-mediated target $m R N A$ repression}

To conclude, we investigated whether this AUF1-facilitated microRNA transfer paradigm impacts on AGO2let-7 activity. To study the influence of AUF1 on let-7 target mRNA expression, we compared the data sets "total RNA-seq (RNA sequencing) after AUF1 overexpression" (which reached twofold to threefold higher levels than endogenous AUF1) (Yoon et al. 2014) and "mRNAs predicted to be let-7 targets." The abundance of let-7 target mRNAs decreased when each isoform of AUF1 or all isoforms together were overexpressed in HEK293 cells compared with nontarget mRNAs (Supplemental Fig. S4A). Among the AUF1-regulated mRNAs (Yoon et al. 2014), we identified two mRNAs bearing let-7b target sites predicted computationally (TargetScan) and confirmed experimentally (miRTCat) (Fig. 4A; Kim et al. 2013): PDP2 and POLR2D mRNAs, encoding pyruvate dehydrogenase phosphatase catalytic subunit 2 and polymerase RNA II polypeptide D, respectively. RIP experiments could not be performed for individual AUF1 isoforms because each isoform lacks a unique epitope, and thus isoform-specific antibodies are not available.

As shown in Figure 4B, silencing AUF1 (all isoforms, as silencing individual isoforms is not technically possible due to the absence of isoform-specific mRNA regions) $(<25 \%$ as shown in Fig. 2A) increased the steady-state abundance of these mRNAs by approximately twofold relative to control cells (Ctrl siRNA). Treatment with actinomycin D to shut off transcription revealed that the halflives of PDP2 and POLR2D mRNAs, as quantified by RT-qPCR analysis (Fig. 4C), increased robustly after silencing AUF1, while the half-life of GAPDH mRNA (a stable mRNA encoding a housekeeping protein) was unaffected by AUF1 levels, indicating that AUF1 selectively lowered the stabilities of PDP2 and POL2D mRNAs. We also found that the half-lives of PDP2 and POL2D mRNAs increased after AGO2 silencing and decreased after let-7b overexpression and that joint silencing of AGO2 and AUF1 further increased the levels of these shared targets (Supplemental Fig. S4B,C). 
To study this interaction by using a different approach, HeLa cells were transfected with biotinylated precursor let-7b (pre-let-7b), and, following lysis, streptavidin beads that pulled down let-7b were found enriched in PDP2 and POLR2D mRNAs but not in lysates from cells transfected with nonbiotinylated control let-7b (Fig. 4D). RIP analysis showed that silencing AUF1 decreased the abundance of AGO2-PDP2 mRNA and AGO2-POLR2D mRNA complexes (Fig. 4E), supporting the observation that let-7b overexpression prevented the rise in PDP2 and POL2RD mRNAs achieved by silencing AUF1 (Fig. 4B). Additional reporter analysis using the firefly luciferase coding sequence with the POLR2D 3' UTR (inserted into the parent vector pMirTarget) revealed that AUF1 silencing increased the stability of the reporter mRNA, while a mutant reporter without the let- $7 \mathrm{~b}$ seed sequence was resistant to changes in AUF1 abundance (Fig. 4F). Collectively, these data indicate that AUF1 can enhance the function of let-7b-AGO2 in lowering the stabilities of PDP2 and POLR2D mRNAs.

\section{Implications of AUF1-facilitated AGO2-let-7b complex formation and function}

Numerous AUF1-mRNA interactions have been studied using a variety of in vitro and in vivo methods (Zucconi et al. 2010; Yoon et al. 2014). Our findings show that AUF1 can also bind some microRNAs (Fig. 1); AUF1 p37 in particular showed strikingly high affinity for let-7b. Indeed, the association of AUF1 and let-7b $(\sim 5.9 \mathrm{nM})$ is in the range of affinity of AGO2 for microRNAs $\left(K_{\mathrm{d}}=10\right.$ $80 \mathrm{nM}$ ) (Tan et al. 2009). Together with the finding that HuR binds let-7b and let-7i with comparable affinities $\left(K_{\mathrm{d}}=1.4\right.$ and $9.6 \mathrm{nM}$, respectively) (Yoon et al. 2013), it is possible that other RBPs are also functionally linked with AGO2 and perhaps are linked more broadly with other AGO proteins.

Possible mechanisms by which RBPs influence AGO2 function include transferring of microRNAs from the RBPs to AGO2, RBPs usurping AGO2 in the RISC, and RBPs competing with AGO2 for binding to microRNAs after microRNA maturation by Dicer in nucleus. In this regard, it is important to mention recent evidence that only a fraction of mature microRNAs, possibly as little as $10 \%$, is bound to AGO proteins, while most microRNAs are bound to mRNAs in the absence of AGO proteins (Janas et al. 2012). The model in which AUF1 can facilitate the transfer of microRNAs to AGO2 is supported by the single-molecule-binding data (Fig. 3; Supplemental Fig. S32), which substantiates a role for AUF1 as an RBP that can "capture" a mature microRNA such as let-7b (perhaps thanks to its high affinity, $5.9 \mathrm{nM}$ ) and "present" or "transfer" microRNAs to AGO2. According to this mode of action, we propose that AUF1 facilitates the formation of AGO2-let-7 complexes (Fig. 4G) and the subsequent targeting of transcripts such as PDP2 and POLR2D mRNAs, which bear let-7 sites. Establishing whether this function is also elicited by other AUF1-bound microRNAs will require further investigation. Future studies should also examine whether the loading of microRNAs onto AGO2 is facilitated more broadly by other RBPs, a feature that would expand the ever-increasing functions of ribonucleoprotein complexes.

\section{Materials and methods \\ Cell culture, transfection, siRNAs, microRNAs, and plasmids}

Human HeLa cells and MEFs were cultured in DMEM (Invitrogen) supplemented with $10 \%(\mathrm{v} / \mathrm{v})$ FBS and antibiotics. To measure mRNA stability, HeLa cells were treated with $2.5 \mu \mathrm{g} / \mathrm{mL}$ actinomycin D for varying time periods, whereupon total RNA was extracted and measured by RT-qPCR analysis to determine their half-lives $\left(t_{1 / 2}\right.$, the time needed for each transcript to reach $50 \%$ of its original abundance). Cells were transfected (Lipofectamine 2000, Invitrogen) with $20 \mathrm{nM}$ siRNAs (control [UUCUCCGAACGUGUCACGUdTdT] or directed to AUF1 [AAGATCCTATCACAGGG CGAT]) or $10 \mathrm{nM}$ pre-let-7b (Ambion). AGO2 siRNA was purchased from Santa Cruz Biotechnology. Plasmids that expressed Flag-tagged AUF1 were described previously (Sarkar et al. 2003) and were transfected at $1-2 \mu \mathrm{g} / \mathrm{mL} \quad(\mathrm{pcDNA}$ and pcDNA-AUF1). Cells were typically analyzed $48 \mathrm{~h}$ after transfection. 


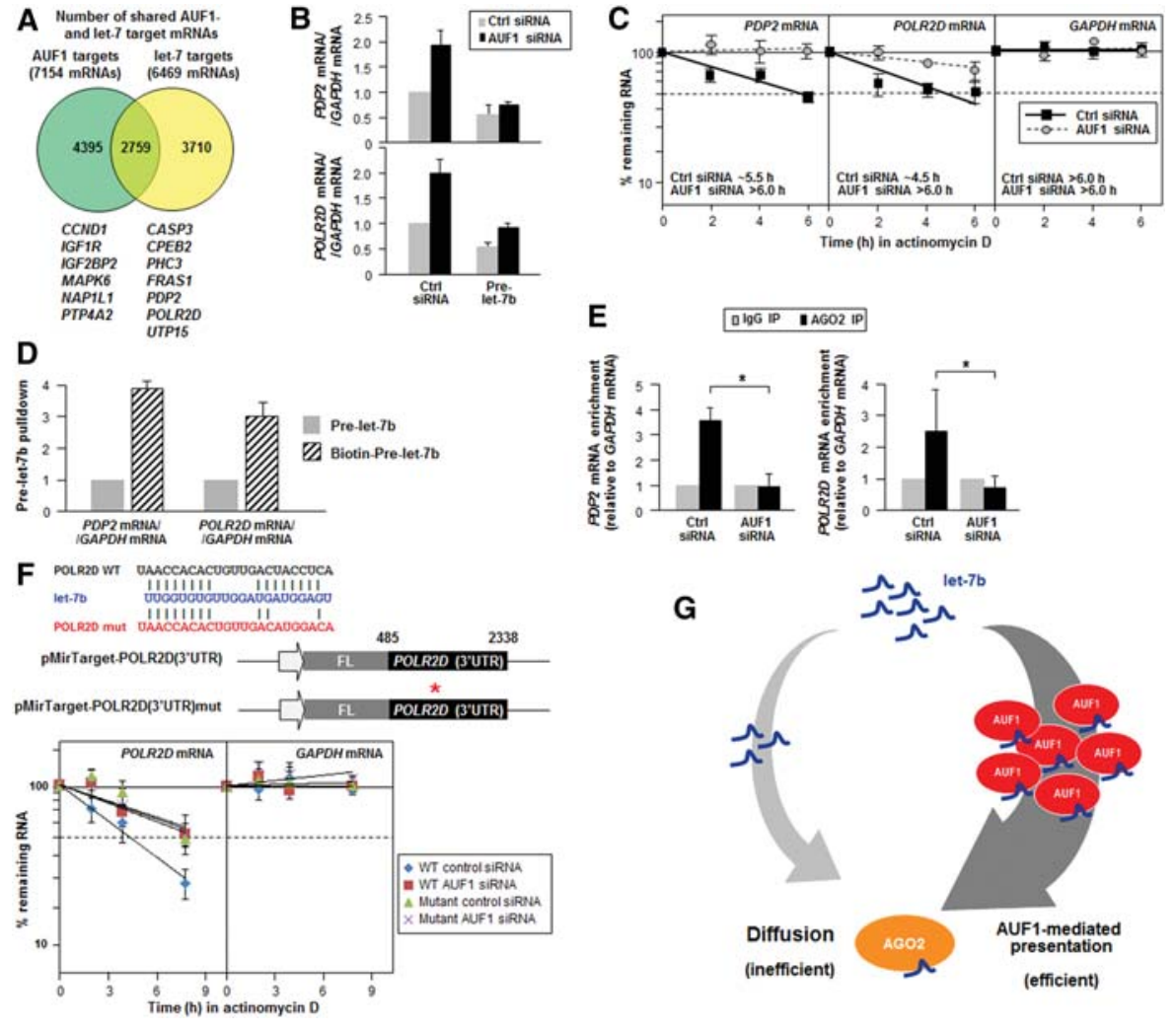

Figure 4. AUF1 cooperates with AGO2-let-7 in the decay of shared target mRNAs. $(A)$ Overlap of AUF1 and let-7 target transcripts and list of representative 13 shared mRNAs. $(B)$ Forty-eight hours after silencing AUF1 or overexpressing let-7b, the abundance of PDP2 and POLR2D mRNAs was assessed by RT-qPCR analysis. $(C)$ Forty-eight hours after transfecting HeLa cells with the siRNAs indicated, the half-lives $\left(t_{1 / 2}\right)$ of PDP2 mRNA, POLR2D mRNA, and GAPDH mRNA (a control stable mRNA encoding a housekeeping protein) were quantified by measuring the time required for reducing transcript levels to $50 \%$ of their original abundance after adding actinomycin D. $(D)$ Forty-eight hours after transfecting HeLa cells using precursor let- $7 \mathrm{~b}$ (pre-let-7b) with or without a biotin label, the association of PDP2 and POLR2D mRNAs with let-7b was assessed by analysis of the RNA present in the pull-down using streptavidin beads; mRNAs were measured by RT-qPCR analysis. (E) Forty-eight hours after transfecting HeLa cells with control (Ctrl) siRNA or AUF1 siRNA, the association of PDP2 and POLR2D mRNAs with AGO2 was assessed by RIP and RT-qPCR analysis; enrichments in each immunoprecipitation was normalized to GAPDH mRNA levels. (F) Fortyeighty hours after transfecting HeLa cells with luciferase reporter constructs (shown at the top) and the siRNAs indicated, the half-lives of wild-type reporter mRNA [FL-POLR2D $\left.\left(3^{\prime} U T R\right)\right]$ and the mutant reporter mRNA [FL-POLR2D( $3^{\prime}$ UTR)mut] as well as the stable control transcript GAPDH mRNA were quantified by measuring their abundance as explained in $C$. (G) Proposed model of AUF1 function in capturing a mature microRNA (let-7) and transferring it to AGO, facilitating the formation of AGO2-microRNA complexes. Data in $B-F$ are the means \pm SD from three independent experiments. $\left({ }^{*}\right) P<0.05$.

\section{Western and Northern blot analysis}

Whole-cell lysates prepared in RIPA buffer were separated by SDSpolyacrylamide gel electrophoresis (SDS-PAGE) and transferred onto nitrocellulose membranes (iBlot Stack, Invitrogen). Primary antibodies recognizing Actin, AGO2, and AUF1 were from Santa Cruz Biotechnology, Abcam, and Millipore, respectively. HRP-conjugated secondary antibodies were from GE Healthcare. Northern blot analysis was performed as described (Supplemental Material; Yoon et al. 2010).

\section{Anisotropy analysis}

Recombinant His-tagged AUF1 or mutant AUF1 was generated in Escherichia coli and purified as described previously (Zucconi et al. 2010). AUF1 binding to $3^{\prime}$-fluorescein-tagged let-7b, miR-21, or miR-130b (Sigma) was evaluated by changes in the fluorescence anisotropy of the tagged microRNA substrates as a function of protein concentration essentially as described (Supplemental Material; Zucconi et al. 2010).

\section{RIP analysis}

For immunoprecipitation of endogenous RNP complexes (RIP analysis) (Keene et al. 2006; Yoon et al. 2012b) from whole-cell extracts, cells were lysed in $20 \mathrm{mM}$ Tris-HCl (pH 7.5), $100 \mathrm{mM} \mathrm{KCl}, 5 \mathrm{mM} \mathrm{MgCl} 2$, and $0.5 \% \mathrm{NP}-40$ for $10 \mathrm{~min}$ on ice and centrifuged at $10,000 \mathrm{~g}$ for $15 \mathrm{~min}$ at $4^{\circ} \mathrm{C}$. The supernatants were incubated with protein A-Sepharose beads coated with antibodies that recognized AGO2 (Abcam) or AUF1 (Millipore) or with control IgG (Santa Cruz Biotechnologyl for $1 \mathrm{~h}$ at $4^{\circ} \mathrm{C}$. After the beads were washed with NT2 buffer (50 mM Tris- $\mathrm{HCl}$ at $\mathrm{pH} 7.5,150 \mathrm{mM} \mathrm{NaCl}$, $1 \mathrm{mM} \mathrm{MgCl}_{2}, 0.05 \%$ NP-40), the complexes were incubated with $20 \mathrm{U}$ of RNase-free DNase I for $15 \mathrm{~min}$ at $37^{\circ} \mathrm{C}$ and further incubated with $0.1 \%$ SDS and $0.5 \mathrm{mg} / \mathrm{mL}$ Proteinase $\mathrm{K}$ for $15 \mathrm{~min}$ at $55^{\circ} \mathrm{C}$ to remove DNA and proteins, respectively. The RNA isolated from the immunoprecipitation materials was further assessed by RT-qPCR analysis using the primers listed (Supplemental Table S1). Normalization of RIP results was carried out by quantifying in parallel the relative levels of U6 snRNA (for RIP analysis of small RNAs) and GAPDH mRNA (for RIP analysis of longer RNA) in each immunoprecipitation sample. These abundant RNAs were nonspecific contaminants present in the immunoprecipitation components (such as the microfuge tube and beads).

\section{RNA analysis}

Trizol (Invitrogen) was used to extract total RNA, and acidic phenol (Ambion) was used to extract RNA for RIP analysis (Keene et al. 2006; Yoon et al. 2012b). Reverse transcription was performed using random hexamers, reverse transcriptase (Maxima, Thermo Scientific), real-time quantitative qPCR using gene-specific primers (Supplemental Table S4), and SYBR Green master mix (Kapa Biosystems) using an Applied Biosystems 7300 instrument. MicroRNA quantitation was performed after RNA extraction from immunoprecipitated samples /with anti-AGO2 or control IgG), polyadenylation (System Biosciences QuantiMiR kit), and hybridization with oligo-dT adaptors. After reverse transcription, cDNAs were quantitated by qPCR with microRNA-specific primers or with primers to detect the control transcript $U 6$ along with a universal primer.

\section{RNA EMSA}

Complex formation between AUF1 and selected microRNA substrates was monitored by RNA EMSA essentially as described (Supplemental Material; Wilson and Brewer 1999). MicroRNA substrates were radiolabeled with $\left[\gamma^{32} \mathrm{P}\right] \mathrm{ATP}$ by T4 polynucleotide kinase to a specific activity of $3 \times$ $10^{3}$ to $5 \times 10^{3} \mathrm{cpm} / \mathrm{fmol}$ and incubated with recombinant purified proteins at different concentrations (up to $1 \mu \mathrm{M}$ ).

\section{Single-molecule-binding analysis}

Quartz slides and cover slips were cleaned using piranha solution (concentrated sulfuric acid/hydrogen peroxide [30\%; 3:1]) for $20 \mathrm{~min}$ and then coated with a 40:1 mixture of polyethylene glycol (m-PEG succinimidyl 
valerate; MW 5000, Laysan Bio, Inc.) and biotinylated polyethylene glycol (biotin-PEG-SC; MW 5000, Laysan Bio, Inc.) to minimize nonspecific binding. The detection chamber consisted of a quartz slide and a coverslip connected to a syringe pump (PHD 2000, Harvard Apparatus) via polyethylene tubing (PE 50; $\mathrm{BD}$ ) for buffer exchange during measurement. For surface immobilization of human AGO2 (hAGO2), streptavidin, anti-His antibodies (6xHis tag antibody biotin-conjugated) (Rockland Immunochemicals, Inc.), and hAGO2 (Sino Biological, Inc.) were successively injected into the detection chamber. Single-molecule fluorescence images were taken using an internal reflection fluorescence microscope. Cy5 was excited by a red laser (Exceisior-635-5c, Spectra Physics) and imaged on an electronmultiplying charge-coupled device (IXON DV597ECS-BV, Andor Technology). The experiments were performed at $37^{\circ} \mathrm{C}$ in $20 \mathrm{mM}$ Tris- $\mathrm{HCl}$ buffer (pH 8.0) with $135 \mathrm{mM} \mathrm{KCl}, 1 \mathrm{mM} \mathrm{MgCl}$, and an oxygen scavenger system ( $1 \mathrm{mM}$ Trolox, $1 \mathrm{mg} / \mathrm{mL}$ glucose oxidase, $0.04 \mathrm{mg} / \mathrm{mL}$ catalase, $0.4 \%[\mathrm{w} / \mathrm{v}]$ glucose [all from Sigma-Aldrich]). For the RNase digestion experiment, Cy5-let-7b RNA was incubated with $1 \mathrm{U}$ of RNase A (Invitrogen) in $20 \mathrm{mM}$ Tris- $\mathrm{HCl}$ buffer (pH 7.5) with $135 \mathrm{mM} \mathrm{KCl}$ and $1 \mathrm{mM} \mathrm{MgCl}_{2}$ for 5 min at $37^{\circ} \mathrm{C}$ before injection.

\section{Acknowledgments}

We thank R.J. Schneider for providing reagents and information, and J.A. Steitz for critical reading of the manuscript. J.-H.Y., S.D., K.A., X.Y., K.G.B., J.L.M., W.H.W., and M.G. were supported by the National Institute on Aging-Intramural Research Program (National Institutes of Health). E.J. F.W., B.E.Z., and G.M.W. were supported by American Heart Association grant 11PRE6900008 and National Institutes of Health grant R01 CA102428. M.H. was supported by the National Institute of Arthritis and Musculoskeletal and Skin Diseases-Intramural Research Program (National Institutes of Health) and the Charles H. Revson Foundation. T.T. was supported by the Howard Hughes Medical Institute, the Starr Cancer Foundation, and the National Institutes of Health. M.H.J. and S.H. were supported by Creative Research Initiatives (Physical Genetics Laboratory, 2009-0081562) of the National Research Foundation of Korea. Y.M.S. and J.-J.S. were funded by grants NRF-M1AXA002-2011-0031416, 2011-0031955, and N1014002 from the Korean Ministry of Science, ICT, and Future Planning. J.-H.Y., M.H.J., E.J.F.W., S.D., M.H., B.E.Z., K.A., X.Y., Y.M.S., J.L.M., W.H.W., J.-J.S., and K.G.B. designed and performed experiments. J.-H.Y., M.G., G.M.W., M.H., S.H., and T.T. prepared the manuscript. The authors discussed the results and implications throughout.

\section{References}

Ambros V. 2004. The functions of animal microRNAs. Nature 431: 350-355.

Bartel DP. 2004. MicroRNAs: genomics, biogenesis, mechanism, and function. Cell 116: 281-297.

Chang N, Yi J, Guo G, Liu X, Shang Y, Tong T, Cui Q, Zhan M, Gorospe M, Wang W. 2010. HuR uses AUF1 as a cofactor to promote p16INK4 mRNA decay. Mol Cell Biol 30: 3875-3886.

Chekulaeva M, Filipowicz W. 2009. Mechanisms of miRNA-mediated post-transcriptional regulation in animal cells. Curr Opin Cell Biol 21: 452-460.

Filipowicz W. 2005. RNAi: the nuts and bolts of the RISC machine. Cell 122: $17-20$.

Glisovic T, Bachorik JL, Yong J, Dreyfuss G. 2008. RNA-binding proteins and post-transcriptional gene regulation. FEBS Lett 582: 1977-1986.

Hafner M, Landthaler M, Burger L, Khorshid M, Hausser J, Berninger P, Rothballer A, Ascano M Jr, Jungkamp AC, Munschauer M, et al. 2010. Transcriptome-wide identification of RNA-binding protein and microRNA target sites by PAR-CLIP. Cell 141: 129-141.

Hafner M, Lianoglou S, Tuschl T, Betel D. 2012. Genome-wide identification of miRNA targets by PAR-CLIP. Methods 58: 94-105.
Janas MM, Wang B, Harris AS, Aguiar M, Shaffer JM, Subrahmanyam YV Behlke MA, Wucherpfennig KW, Gygi SP, Gagnon E, et al. 2012. Alternative RISC assembly: binding and repression of microRNA-mRNA duplexes by human Ago proteins. RNA 18: 2041-2055.

Jo MH, Shin S, Jung SR, Kim E, Song JJ, Hohng S. 2015. Human Argonaute 2 has diverse reaction pathways on target RNAs. Mol Cell 59: 117-124.

Joshua-Tor L. 2006. The Argonautes. Cold Spring Harb Symp Quant Biol 71: 67-72.

Keene JD, Komisarow JM, Friedersdorf MB. 2006. RIP-ChIP: the isolation and identification of mRNAs, microRNAs and protein components of ribonucleoprotein complexes from cell extracts. Nat Protoc 1: 302-307.

Kim KK, Ham J, Chi SW. 2013. miRTCat: a comprehensive map of human and mouse microRNA target sites including non-canonical nucleation bulges. Bioinformatics 29: 1898-1899.

Panda AC, Abdelmohsen K, Yoon JH, Martindale JL, Yang X, Curtis J, Mercken EM, Chenette DM, Zhang Y, Schneider RJ, et al. 2014. RNA-binding protein AUF1 promotes myogenesis by regulating MEF2C expression levels. Mol Cell Biol 34: 3106-3119.

Pont AR, Sadri N, Hsiao SJ, Smith S, Schneider RJ. 2012. mRNA decay factor AUF1 maintains normal aging, telomere maintenance, and suppression of senescence by activation of telomerase transcription. Mol Cell 47: 5-15.

Salomon WE, Jolly SM, Moore MJ, Zamore PD, Serebrov V. 2015. Singlemolecule imaging reveals that Argonaute reshapes the binding properties of its nucleic acid guides. Cell 162: 84-95.

Sarkar B, Xi Q, He C, Schneider RJ. 2003. Selective degradation of AU-rich mRNAs promoted by the p37 AUF1 protein isoform. Mol Cell Biol 23: 6685-6693.

Tan GS, Garchow BG, Liu X, Yeung J, Morris JP IV, Cuellar TL, McManus MT, Kiriakidou M. 2009. Expanded RNA-binding activities of mammalian Argonaute 2. Nucleic Acids Res 37: 7533-7545.

White EJ, Brewer G, Wilson GM. 2013. Post-transcriptional control of gene expression by AUF1: mechanisms, physiological targets, and regulation. Biochim Biophys Acta 1829: 680-688.

Wilson GM, Brewer G. 1999. Identification and characterization of proteins binding A + U-rich elements. Methods 17: 74-83.

Wu X, Chesoni S, Rondeau G, Tempesta C, Patel R, Charles S, Daginawala N, Zucconi BE, Kishor A, Xu G, et al. 2013. Combinatorial mRNA binding by AUF1 and Argonaute 2 controls decay of selected target mRNAs. Nucleic Acids Res 41: 2644-2658.

Yoon JH, Choi EJ, Parker R. 2010. Dcp2 phosphorylation by Ste20 modulates stress granule assembly and mRNA decay in Saccharomyces cerevisiae. J Cell Biol 189: 813-827.

Yoon JH, Srikantan S, Gorospe M. 2012a. MS2-TRAP (MS2-tagged RNA affinity purification): tagging RNA to identify associated miRNAs. Methods 58: 81-87.

Yoon JH, Abdelmohsen K, Srikantan S, Yang X, Martindale JL, De S, Huarte M, Zhan M, Becker KG, Gorospe M. 2012b. LincRNA-p21 suppresses target mRNA translation. Mol Cell 47: 648-655.

Yoon JH, Abdelmohsen K, Kim J, Yang X, Martindale JL, Tominaga-Yamanaka K, White EJ, Orjalo AV, Rinn JL, Kreft SG, et al. 2013. Scaffold function of long non-coding RNA HOTAIR in protein ubiquitination. Nat Commun 4: 2939.

Yoon JH, De S, Srikantan S, Abdelmohsen K, Grammatikakis I, Kim J, Kim KM, Noh JH, White EJF, Martindale JL, et al. 2014. PAR-CLIP analysis uncovers AUF1 impact on target RNA fate and genome integrity. Nat Commun 5: 5248.

Zucconi BE, Wilson GM. 2011. Modulation of neoplastic gene regulatory pathways by the RNA-binding factor AUF1. Front Biosci 16: 23072325 .

Zucconi BE, Ballin JD, Brewer BY, Ross CR, Huang J, Toth EA, Wilson GM. 2010. Alternatively expressed domains of AU-rich element RNA-binding protein 1 (AUF1) regulate RNA-binding affinity, RNA-induced protein oligomerization, and the local conformation of bound RNA ligands. J Biol Chem 285: 39127-39139. 


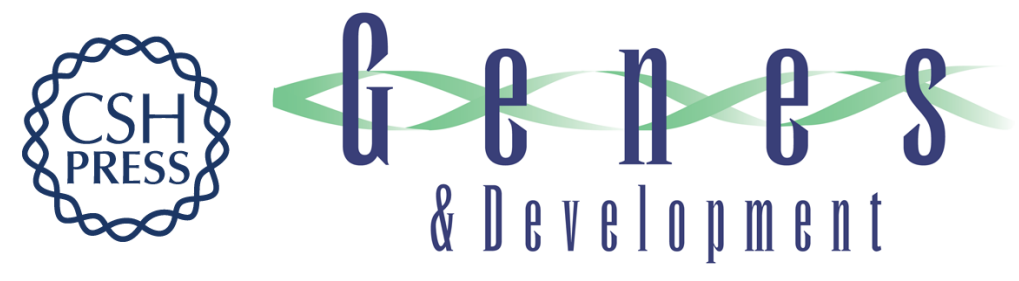

\section{AUF1 promotes let-7b loading on Argonaute 2}

Je-Hyun Yoon, Myung Hyun Jo, Elizabeth J.F. White, et al.

Genes Dev. 2015, 29:

Access the most recent version at doi:10.1101/gad.263749.115

Supplemental Material

References

Creative Commons License

Email Alerting Service
http://genesdev.cshlp.org/content/suppl/2015/08/07/29.15.1599.DC1

This article cites 28 articles, 7 of which can be accessed free at: http://genesdev.cshlp.org/content/29/15/1599.full.html\#ref-list-1

This article is distributed exclusively by Cold Spring Harbor Laboratory Press for the first six months after the full-issue publication date (see

http://genesdev.cshlp.org/site/misc/terms.xhtml). After six months, it is available under a Creative Commons License (Attribution-NonCommercial 4.0 International), as described at http://creativecommons.org/licenses/by-nc/4.0/.

Receive free email alerts when new articles cite this article - sign up in the box at the top right corner of the article or click here.

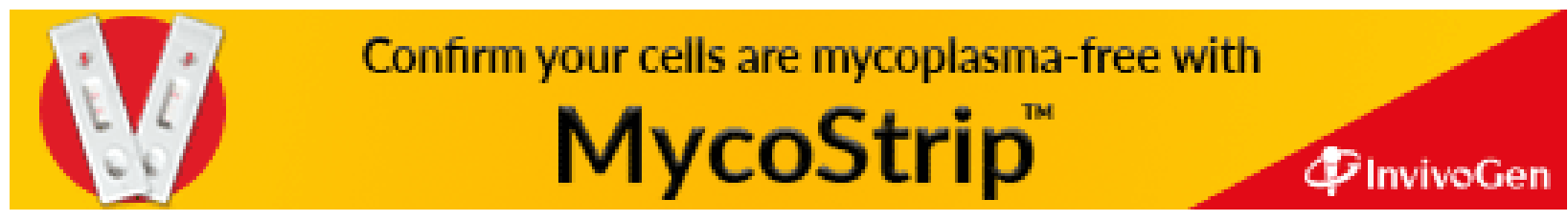

